\title{
Biomarkers and their dependence on well-reported antibodies
}

Jan Voskuil is the Chief Scientific Officer at antibody manufacturer Everest Biotech in Oxfordshire, UK. After specializing in prokaryotic cell biology through his PhD program in Amsterdam, The Netherlands and a postdoctorate position at Stanford (CA, USA), he switched to the science of neurodegenerative diseases at Oxford, UK through postdoctorate positions at Dunn School of Pathology and at MRC and through a leading position at the Alzheimer drug discovery company Synaptica. He subsequently gained experience in a Good Laboratory Practice-regulatory environment in contract research organization companies both in Oxfordshire and Cambridgeshire, validating assays in Flow Cytometry and ELISA platforms and writing standard operating procedures. His extensive experience with generating and characterizing monoclonal and polyclonal antibodies in combination with accrued knowledge on most immunoassays in academic and commercial environments made him the ideal candidate to take charge in putting Everest Biotech on the global map by ever raising the quality and size of its catalog and by delivery of adequate technical support. As a result, Everest antibodies are currently part of most globally well-known catalogs, and its products are increasingly recognized as useful alternatives to unfit monoclonal antibodies.

Keywords: antibodies $\bullet$ biomarkers $\bullet$ biomedical research $\bullet$ validation

\section{Q Can you provide us with a brief} overview of your career to date?

I completed my PhD in Amsterdam (The Netherlands) before taking up postdoctorate positions at Stanford (CA, USA) and Oxford (UK). I switched from academia to industry through a leading position at the CNS drug discovery company Synaptica. I subsequently gained experience in Good Laboratory Practice-regulatory environments at contract research organizations in Oxfordshire and Cambridgeshire (UK). I hope that my combined academic and commercial background together with my technical and people skills as the Chief Scientific Officer have helped put Everest Biotech (UK) on the global map with the highest quality standard. Its antibodies are increasingly recognized as alternatives to unfit monoclonal antibodies.

\section{Q What inspired you to work in the} field of commercial antibodies?

As a scientist, I have always been keen to make a difference and needed to find my niche. It became clear through my adventures in academia that I was not to become a principle investigator. I did, however, learn a lot about the difficulties of laboratory work on a dayto-day basis. From a provider's angle, I developed essential communication skills; not as a salesperson, but as a scientist with empathy for my colleagues in the laboratory. As a result, I began building a reputation as an antibody provider with a genuine interest in helping scientists develop their research, instead of prioritizing short-term financial gain at the expense of customers. This was 10 years ago, when the majority of commercial antibodies on catalogs were not trustworthy and customers took a gamble when buying. We have come a long

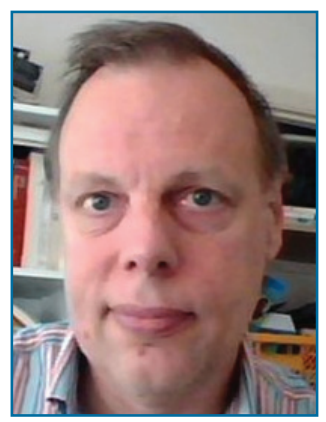

Jan Voskuil

Everest Biotech Ltd, Cherwell Innovation Centre 77 Heyford Park, Upper Heyford, Oxford, OX25 5HD, UK

voskuil@everestbiotech.com

Future
Medicine $\mathrm{fS}$ 
way since then and we, at Everest, are proud of our role in setting a trend of offering ever-increasing quality antibodies to the market. Consequently, scientists now have much less to worry about in terms of purchasing ineffective commercial antibodies. Although much work remains, most providers now thankfully issue refunds if an antibody does not conform according to its specifications on the datasheet within the laboratory. The contribution of high-quality antibodies to the progress of biomedical sciences should not be underestimated.

\section{Q What would you describe as the biggest} achievement of your career to date?

The strategy described above has led to a dramatic increase in sales from our Everest Biotech catalog. Primarily through vendors rebranding our quality products as their own, but increasingly through direct sales to assay developer companies who now recognize that the monoclonal antibodies they previously used do not always meet the requirements. Everest Biotech products offer cost-effective alternative. The reputation I have built should be regarded as my greatest achievement because it has made Everest Biotech an increasingly profitable company, whose growth continued through the recent recession and is getting stronger year on year.

\section{Q Are there any developments within Everest} Biotech, recent or forthcoming, that have you particularly excited?

Yes, since our catalog obtained a critical mass a few years ago, we inevitably started to manufacture some extra products against proteins that we had developed antibodies to in the past. This now gives us the opportunity to offer pairs of antibodies as separate items for the catalog, for example, for use in sandwich-type ELISA. We are currently in the middle of generating the quality control so that we are able to launch this new product line by the end of this financial year, if not sooner. Key is that we have thoroughly established that our peptide goat antibodies show sufficient batch-tobatch consistency to offer our products as commercial kits. In fact, our products have already been included in commercial kits offered by other vendors. What excites me most is the new prospect of immortalizing our bestselling products without having to change the catalog number! This means we will eventually have unlimited production without goats involved, thus gaining access to the diagnostic market. This is the latest development to be rolled out, so watch this space.

Q You often speak of lack of trust, in regards to antibodies, within the medical community, why is this the case?
We should make a distinction between therapeutic and diagnostic antibodies on the one hand and research antibodies on the other. The former two undergo validation according to regulatory requirements so that, before such an antibody is being used in the clinic, it has been thoroughly checked and double checked. However, research antibodies do not undergo such rigorous validation process. This would be impractical because each scientist would need the antibody only for their own purpose.

Despite our wishes, the majority of research antibodies do not work in every type of immunoassay. Some will work in western blot and possibly in immunoprecipitation, while others will work in immunohistochemistry and immunocytochemistry. There are catalogs completely dedicated to flow cytometry. Some of these antibodies are fit for other purposes while others are not. This reality causes some confusion as not all scientists wish to accept this fact. There are still many scientists that first employ a western blot to test antibodies for specificity, even if the antibody requires a different platform. It is not generally accepted that the conditions in different immunoassays will determine the performance of the antibody so, as a result, many are dismissed following failure in western blot, before the recommended validation is undertaken. In addition, many scientists are unaware that identical antibodies may be present in separate catalogs.

I wrote a paper addressing the different factors that cause mistrust of commercial research antibodies [1] and I hope that it helps the science community gain more trust in commercial research antibodies. There are no rogue antibody vendors anymore, at least not that I know of, and negative feedback is now valued as highly as positive feedback. All antibody vendors benefit from timely information regarding cases of nonconformity so that they can take appropriate action.

\section{Q How does this affect the clinical validation} of biomarkers \&, in turn, the adoption of personalized medicine?

There are biomarker databases with thousands of entries, yet the vast majority are never applied in the clinic. I have spent considerable time on protein biomarkers, which form the bulk of the Everest Biotech pipeline. It has become clear that the majority of biomarkers suffer from a lack of evidence regarding their utility. There are several reasons for this dearth of evidence, which I have summarized in the following paper [2].

In recent months a spotlight has been shone on the issues surrounding the reproducibility of published biomedical research data. One allegation was that research antibodies were to blame. If it was only 
that simple! Most biomarkers, apart from protein biomarkers, do not actually depend on antibodies for their detection. When they do, however, there may be inconsistencies between results depending on the antibody used to detect a biomarker. Poor reporting of the antibody used can prevent others from reproducing the data, for example when the antibody was merely specified as a mouse antibody from vendor $\mathrm{X}$ without mentioning the catalog number. When vendor $\mathrm{X}$ has three mouse antibodies against this biomarker on its catalog, which one was used in the publication?

A specific antibody is defined by its epitope: the part of the protein the antibody binds to. Nonetheless, many monoclonal antibodies are used without the epitope being known. This lack of knowledge introduces the risk of cross-reactivity to other related proteins sharing this epitope. A historic example are the HER 2 antibodies used in the diagnosis of HER2-type breast cancer; from the three commercially available products (all monoclonal antibodies, all rigorously validated before being allowed on the market as a diagnostic), only one turned out to be HER2-specific, and the other two cross-reacted to HER4. This is what happens when antibodies are used without the epitope having been characterized! Had it been known, this cross-reaction could have been predicted.

Clearly for stratified medication or personalized medicine, we first need to have standards in the detection of the biomarkers involved.

\section{Q How can we overcome these challenges?}

I do not have all the answers, but we can make a start with a few measures. There are already discussions on the internet and in editorials on whether to make it compulsory for investigators to report the catalog numbers for antibodies employed in published data. This would facilitate the reproduction of published data generated through the use of antibodies.

Additionally, as discussed previously, it would benefit the biomedical community to stick to wellcharacterized antibodies with known epitope/antigen sequences. This would rule out many monoclonal antibodies and all nonpeptide generated polyclonal antibodies. This suggestion will be deemed controversial, however, because monoclonal antibodies with unknown epitopes have been used for many years as the gold standard and the data generated by them cannot be dismissed. Instead of dismissing all such data, I believe we should attempt to identify the epitopes of previously used antibodies. Of course there are those few antibodies thought to recognize a structural epitope, which is difficult to identify. I acknowledge that these challenges will be difficult to overcome.
Q Global transparency \& data sharing is clearly a key point here, is Everest Biotech undertaking any work toward this goal?

We have consistently published the sequences of the immunizing peptides for each of our products. This creates clarity, and scientists can determine for themselves whether our product will likely be fit for their work by assessing this sequence. I have seen other vendors offering peptide antibodies without displaying the immunizing peptide sequence, relying on the scientist to contact them and ask for it. I suspect that such products have been generated from the same peptide but in a different species than the original product. Vendors would do this if they buy and sell great quantities of an original antibody and create their own version with the aim of replacing the original and increasing their profit margins. However, this is ineffective as it takes years for an antibody to become established in the market through use in scientific publications and, by then, customers are not interested in a similar antibody from a different species. The key is to be upfront with customers. We have our products on many large catalogs and we are happy for them to rebrand our products and to sell them as their own. However, there is no point in deceiving the scientists. It has not worked in the past and it will never work. Transparency in the commercial antibody field remains the only way to long-term business success.

When it comes to biomedical research, progress has been hampered by the lack of scientific data sharing. Similar to the antibody business, openly sharing data (both for fundamental biomedical science and for clinical science) will minimize needless repetitions of experiments that do not produce useful results. I know that the pharmaceutical industry has traditionally been very secretive about their data due to concerns over intellectual property. However, new medications drive their business and these cannot be developed without the proper science in place. So, there is no reason to be secretive about the science, and in fact the most successful businesses do publish their science in the official scientific journals anyway! The main trouble is that negative data are not being shared.

\section{Q How do you see your field progressing in the next 5-10 years?}

There will likely be a full integration of fundamental science (using proper research antibodies) with both the diagnostic and pharmaceutical industries. The current challenge in the biomedical world is to understand the etiology of complex diseases like cardiovascular diseases, cancers, dementias, obesity and depression. Unlike in the first half of the previous century when we successfully identified pathological micro-organisms and viruses and subsequently found 
remedies to them, progress in regards to the above ailments is slow. I believe that advances will come through increased investigation in fundamental biological science. Our efforts so far have brought to light different approaches like genomics, proteomics, epigenetics and metabolomics, but none of these are likely to produce definitive answers on their own. We need to progress on all fronts and get them all integrated. This will ultimately result in a highly specific multidimensional profile, possibly as personal as a fingerprint. With all these data, we will be able to find new clues on the early stages of these terrible diseases and new candidate therapies, with higher efficacy and fewer side effects, will be easier to identify.

\section{References}

1 Voskuil J. Commercial antibodies and their validation. F1000 Res. doi:10.12688/f1000research.4966.2 (2014) (Epub ahead of print).

\section{Disclaimer}

The opinions expressed in this interview are those of the interviewee and do not necessarily reflect the views of Future Medicine Ltd.

\section{Financial \& competing interests disclosure}

J Voskuil has no relevant affiliations or financial involvement with any organization or entity with a financial interest in or financial conflict with the subject matter or materials discussed in the manuscript. This includes employment, consultancies, honoraria, stock ownership or options, expert testimony, grants or patents received or pending, or royalties.

No writing assistance was utilized in the production of this manuscript.

2 Voskuil J. How difficult is the validation of clinical biomarkers? F1000 Res. doi:10.12688/f1000research.6395.1 (2015) (Epub ahead of print). 